\section{Preserving personal autonomy in a genomic testing era}

To the Editor: We read with great interest the article titled "An Informatics Approach to Analyzing the Incidentalome" by Berg et al. ${ }^{1}$ whose strategy surely is the direction of the future for clinical whole-exome/whole-genome sequencing (WES/WGS), with a focus on automation and reduction of output of uninterpretable results. The Mayo Clinic Center for Individualized Medicine has also been developing an approach with similar bioinformatics strategies, but it differs in the approach to binning. This letter is written to highlight alternative ways to group disorders for purposes of providing clinical choices.

Berg et al. ${ }^{1}$ used three bins to classify genes: Bin $1(n=161)$ genes, deemed to have clinical utility, would be returned to patients by default; Bin 2 genes, having clinical validity but no appreciable clinical utility, were further subdivided into Bin $2 \mathrm{~b}(n=1,798)$ and Bin $2 \mathrm{c}(n=57)$ based on a likely increasing potential for harm due to "their potential to cause psychosocial harm if revealed unexpectedly to an inadequately prepared individual." The genes in Bin $2 \mathrm{c}$ are mostly untreatable neurodegenerative disorders. The authors proposed that "the return of Bin 2 findings be carefully guided by an appropriately qualified clinician, in the context of that individual's personal situation, or not reported at all." Bin 3 contained all other genes whose roles in human disease are undefined. The authors underscored that the binning used in the study is provisional, expecting the final population of bins to change over time.

Even among experts, differences of opinion exist regarding what types of incidental genetic results should be returned. In a pilot study, 16 specialists in clinical/molecular genetics, a group of early adopters of genomic medicine, were asked to score variants in 99 common conditions to return to the ordering physician if discovered incidentally through WES/ WGS. ${ }^{2}$ Results showed imperfect concordance across this relatively homogeneous group of specialists, suggesting to us not that one should try to reach consensus but that we should strive for better ways to let individuals make their own decisions.

The past two decades have provided copious data confirming that there is wide individual variability in interest/desire to know one's genetic risks. Initial concerns of psychological harm were, fortunately, mostly unfounded. A primary lesson learned was that well-prepared patients make decisions for themselves that they can live with comfortably. The Mayo Center for Individualized Medicine has been grappling with development of a strongly patient-centered approach to integration of WES/WGS into clinical practice. Our approach starts with a structured genetic counseling component. We have created tools to assist patients not just in understanding genetics but in being able to articulate and prioritize their personal values and goals for having WES/WGS. These tools evolved out of design-centric ideas from the Mayo Center for Innovation, feedback from a community advisory board of the Mayo Clinic Biobank, and a team of genetic specialists. Use of these tools in the counseling process leads more easily to concrete decisions by the patients regarding opting in/ opting out of learning certain types of information. To link discovered mutations with disease phenotypes, it is common practice to use standard ontologies, such as the OMIM gene numbers. However, there are problems associated with this, because many genes have multiple OMIM phenotype numbers (e.g., FBN1 has seven different phenotypes; several entries on Berg et al.'s lists are phenotype numbers), and many OMIM phenotypes (e.g., retinitis pigmentosa) have multiple genes associated with them. We have developed an in-house system called the Tailored Result Selection Tool (TRuST) for 3,753 phenotypes (3,158 different phenotypes) that includes 2,478 different genes (and growing). All genes/ phenotypes are scored across multiple domains that include a score for "actionability," which our work suggests is the concept that most resonates with people who are deciding about opting in or opting out of results. We do not find that actionability can be meaningfully distilled down to a "yes or no" choice, but rather it occupies a continuum ranging from disorders that are partially preventable or treatable to those diseases for which there is no prevention or treatment available. We do think that with adequate pretest counseling and education, individuals can make good informed decisions on this continuum and that this is preferable to a scenario in which there is no opt-out choice. No one can disagree that knowing of a BRCA1 mutation can lead to many constructive responses, but we all know that there are insightful individuals who, for whatever reason at that moment in their lives, say "Thanks, but no thanks." Preserving that choice is important still.

In addition to using TRuST guidance for framing clinical opt-in/opt-out choices, this system may be useful to researchers and biobanks as they question which results should be offered back to participants and struggle to balance the effort to contact participants with "actionability" and participant expectations.

A 1-10 scale was used initially for the TRuST actionability scores to describe how adequately medical intervention restored or protected optimal health. We compared the published bins ${ }^{1}$ with our scores, which are currently combined into four groups: 
Group A: No specific medical management can be offered to change the most significant, serious, or debilitating aspect of the disease (scores 9-10). Genes in published Bin $2 \mathrm{c}$ scored a mean of 9.3 , suggesting some agreement on lack of treatability. However, we have 1,445 OMIM entities that also have general lack of actionability. Not all are life threatening, but all are untreatable.

Group B: Limited medical management is available, but the most serious aspects of the disease are not improved or fully prevented (scores 6-8).

Group C: Medical interventions are recognized as helpful; however, medical interventions do not eliminate all the medical issues and risks associated with the disease (scores 3-5). Published Bin 1 had a mean score of 3.9, trending toward treatable but also indicating that the actions that can be taken incompletely address some disorders.

Group D: Medical treatments and management for the disease are available that essentially restore ideal health (scores 1-2). Thirty-two entries from Bin 1 were in this category.

Multiple other domains were scored in TRuST, including inheritance, childhood lethality, progressive nature, organ system/function affected (includes dementia), and severity (although it remains to be seen if it can be validated). One might want to know if an untreatable condition is very serious or rather minor, if opting in or opting out of knowing one's status. There are no data to support one classifying system over another, nor have real-world preferences and needs been distilled for clinical WES/WGS. We will share lessons learned as we refine and attempt to validate the TRuST domains to determine what is useful or useless to provide useful choices for our patients.

\section{DISCLOSURE}

The authors declare no conflict of interest.

Noralane M. Lindor, $M D^{1}$, Kiley J. Johnson $C G C^{2}$, Jennifer B. McCormick, $P h D, M P P^{3}$, Eric W. Klee, $P h D^{4}$, Matthew J. Ferber, $P h D^{5}$ and Gianrico Farrugia, $M D^{6}$

\footnotetext{
${ }^{1}$ Department of Health Science Research, Mayo Clinic Arizona, Scottsdale, Arizona, USA; ${ }^{2}$ Center for Individualized Medicine, Mayo Clinic Rochester, Rochester, Minnesota, USA; ${ }^{3}$ Biomedical Ethics, Department of General Internal Medicine, Mayo Clinic Rochester, Rochester, Minnesota, USA; ${ }^{4}$ Biomedical Statistics and Informatics, Department of Health Science Research, Mayo Clinic Rochester, Rochester, Minnesota, USA; ${ }^{5}$ Department of Laboratory Medicine and Pathology, Mayo Clinic Rochester, Rochester, Minnesota, USA; ${ }^{6}$ Center for Individualized Medicine and Division of Gastroenterology, Mayo Clinic Rochester. Rochester, Minnesota, USA. Correspondence:

Noralane M. Lindor (nlindor@mayo.edu)
}

\section{REFERENCES}

1. Berg JS, Adams M, Nassar N, et al. An informatics approach to analyzing the incidentalome. Genet Med 2013;15:36-44.

2. Green RC, Berg JS, Berry GT, et al. Exploring concordance and discordance for return of incidental findings from clinical sequencing. Genet Med 2012;14: $405-410$

doi:10.1038/gim.2013.24

\section{Response to Lindor et al.}

To the Editor: We greatly appreciate the comments of Dr Lindor and colleagues in the letter titled "Preserving Personal Autonomy in a Genomic Testing Era" regarding our approach to managing incidental findings from genome-scale sequencing. ${ }^{2}$ We wholeheartedly agree that a consensus-based approach for determining how to handle the broad spectrum of incidental findings is unlikely to satisfy all constituents in the long term. Likewise, we are in complete agreement that patient preferences should play a central (although not exclusive) role in determining the return of results. Indeed, our "binning" approach attempts to balance the ethical responsibilities of the clinician (such as the duty to warn) with the autonomy of the patients to determine what information they want to know and what information they prefer not to know.

There are certainly many valid approaches to dividing the genome into categories that can be used to manage the return of incidental findings, but we strongly believe that some measure of clinical actionability will be a critical parameter in any successful strategy. In our approach, Bin 1 can be considered the category of incidental information in which the degree of clinical actionability invokes a duty to warn that supersedes patient preferences. Bin 2 contains the bulk of incidental information with limited clinical actionability that some patients may desire to know, whereas others may not, which is the very definition of individual informed decision making. Of course, there will be differing opinions about where to draw the line between Bin 1 and Bin 2, which is essentially the crux of the problem with consensus-based approaches to "binning" the genome. Instead, as pointed out by Lindor and colleagues, ${ }^{1}$ there is a continuum of actionability.

We are therefore intrigued to hear about the efforts at the Mayo Center for Individualized Medicine to develop the Tailored Result Selection Tool with a scoring system for "actionability," and we were gratified to see that our provisional bin assignments correlated reasonably well with the Mayo group's actionability scores. Our group has come to the very same conclusion that a semiquantitative measure is required to score the clinical actionability of gene-phenotype pairs in order to categorize them in a transparent and evidence-based fashion. We have focused on four key components of clinical actionability: (i) the severity of the threat to health for an undiagnosed individual carrying an incidentally identified deleterious allele; (ii) the likelihood that a serious threat will materialize, akin to penetrance; (iii) the effectiveness of interventions at preventing harm from occurring; and (iv) the acceptability in terms of the burdens or risks placed on the individual. These components of actionability have also been adopted as part of an evidencebased framework being developed by the Evaluation of Genomic Applications in Practice and Prevention working group ${ }^{3}$.

Our local "binning" committee is now systematically scoring gene-phenotype pairs much in the same way as described by Lindor and colleagues. ${ }^{1}$ In the process, we have revised the 